EDITORIAL

\title{
Are troponins confusing?
}

\author{
P O Collinson, P J Stubbs
}

\section{The cardiospecificity and sensitivity of cardiac troponin measurement has proved to be a two edged sword for the laboratory and the clinician}

$\mathrm{T}$ he ability to measure the cardiac specific markers, cardiac troponin $\mathrm{T}$ ( $\mathrm{cTnT}$ ) and cardiac troponin I (cTnI) (the cardiac troponins), has produced a paradigm shift in the assessment of patients with suspected acute coronary syndromes (ACS). Elevations of creatine kinase (CK) and its MB isoenzyme (CK-MB) resulting from skeletal muscle damage can now reliably be distinguished from those caused by acute myocardial infarction (AMI). ${ }^{1}$ In the ACS patient, measurement of CTnT and cTnI identified prognostically significant myocardial damage when myocardial infarction was excluded by conventional tests. ${ }^{2}$ This confirmed and extended previous work showing that minor but non-diagnostic elevations of CK-MB in ACS patients indicated myocardial damage and were prognostic. ${ }^{3}$ The evidence that cTnT and cTnI elevations predict prognosis in patients with and without ST elevation ACS is comprehensive and led to the proposed redefinition of AMI. ${ }^{4}$ This recognised cTnT and CTnI measurement as the biochemical diagnostic "gold standard". The role of cardiac biomarkers to guide treatment has also been recognised and incorporated into management guidelines. ${ }^{5}$

\section{INTERPETING TEST RESULTS}

The cardiospecificity and sensitivity of cardiac troponin measurement has proved to be a two edged sword for the laboratory and the clinician. The laboratory must now produce analytically precise and accurate cardiac marker measurements by immunoassay on a 24 hour, seven day a week basis. The individual laboratory methods for cTnI show differing sensitivities and do not give equivalent answers. ${ }^{1}$ The clinician has found that evidence of cardiac damage can be readily (and unsurprisingly) detected in a range of other clinical conditions. ${ }^{67}$ Is this a problem of the test or the clinical interpretation of the test result? There is no evidence that these are "false positive" results. The original suggestion of reexpression of CTnT in skeletal muscle has been refuted $^{89}$ and elevations are prognostic in patients with renal failure. ${ }^{10}$ The advent of more sensitive methods for measurement of cTnI has demonstrated that both cTnT and CTnI are elevated in a range of clinical conditions. What is the difference between cTnT and cTnI measurements compared to the more conventional cardiac biomarkers $\mathrm{CK}$ and CK-MB? What confers the ability to demonstrate cardiac damage outside the traditional ACS population?

Conventional cardiac enzymes have evolved from measurements of aspartate transaminase and lactate dehydrogenase to CK and finally to CK-MB mass. Each change has been accompanied by improved diagnostic sensitivity and an increase in the number of patients with non-Q wave, now called non-ST elevation MI (NSTEMI). All enzyme measurements, including CK-MB mass, have a background level (a noise level) which is represented by the reference interval (normal range). In contrast, the background level of cTnT and cTnI measured by the current assays appears to be undetectable-that is, effectively zero. This means that lesser damage, previously hidden within the background noise of the reference interval for CK$\mathrm{MB}$, is now detected by the presence of CTnT or cTnI.

\section{SUBCLINICAL CARDIAC DAMAGE}

The concept of subclinical but detectable cardiac damage is not new. Right ventricular strain detectable on the ECG occurs with significant pulmonary embolus. The finding of cTnT and CTnI elevations in patients with large pulmonary embolus supports this. ${ }^{11}$ The surprise is the range of conditions where cTnT and cTnI elevation occurs, from the logical, such as myocarditis, to the paradoxical such as chronic renal failure. These elevations not only indicate myocardial damage, but are also prognostic. Experience at St George's hospital is that as many as $30 \%$ of patients admitted to general medical beds (noncardiac care unit admissions) and with elevated cTnT do not have conventional ACS. What should these patients be classified as? Is it AMI? It must be stressed that cTnT and cTnI elevations are specific for cardiac damage, but cardiac damage does not automatically equate to myocardial infarction. Acute myocardial infarction is a clinical diagnosis. Laboratory measurement is required for diagnosis but is not the only arbiter. Although the laboratory can provide a cardiac specific test, it cannot provide a diagnosis specific test.

Abbreviations: ACC, American College of Cardiology, ACS, acute coronary syndromes; $\mathrm{AMI}$, acute myocardial infarction; $\mathrm{CK}$, creatine kinase; $\mathrm{CTnl}$, cardiac troponin I; cTnT, cardiac troponin T; ESC, European Society of Cardiology; $\mathrm{NICl}$, non-ischaemic cardiac injury; NSTEMI, non-ST elevation myocardial infarction; $\mathrm{PICl}$, primary ischaemic cardiac injury; $\mathrm{SICl}$, secondary cardiac ischaemic injury 


\section{PLAQUE RUPTURE}

Acute coronary syndromes mainly arise from the rupture of an atheromatous plaque. Troponin release predominantly occurs due to two mechanisms. Exposure of the plaque core results in platelet activation and aggregation (white thrombus). These aggregates are unstable and produce distal embolisation with micro-infarction and troponin release. ${ }^{12}$ Progression to activation of the clotting cascade results in fibrin clot formation (red thrombus) with vessel occlusion and an area of necrosis in the territory supplied by the artery. The release of cTnT and cTnI in these circumstances occurs due to a temporal event and follows, in the majority of cases, a defined kinetic. Hence, the use of a rise and fall in the American College of Cardiology/European Society of Cardiology (ACC/ESC) guidelines separates cTnT and cTnI elevations caused by ACS, a primary ischaemic cardiac injury (PICI), from those where there is a sustained injury such as myocarditis. This means that a single measurement of cTnT or cTnI is not sufficient and at least two should be carried out, including one on admission, particularly if an early aggressive intervention strategy is considered. ${ }^{5}$ This is less of an issue as the cost of cTnT and cTnI measurements has fallen substantially.

A number of clinical studies have shown that interventions, especially revascularisation, can be guided by troponin measurement. These have used very low cut off values-for
cTnT $0.01 \mu \mathrm{g} / \mathrm{l}$ has been proposed..$^{13}$ It is important to remember that such studies have been performed in highly selected clinical trial populations where the prior probability of ischaemic heart disease approaches $100 \%$. This does not reflect routine clinical practice, where the probability of ischaemic heart disease is significantly lower in unselected acute admissions with chest pain to a typical casualty department. Minor troponin elevations can only be used to guide treatment in the context of suspected PICI.

Elevations of troponin where PICI is not the cause can be divided into two categories: secondary cardiac ischaemia not caused by primary plaque rupture (SICI), and direct myocardial involvement in the primary pathological process (non-ischaemic cardiac injury (NICI)). Troponin release occurring during cardiac procedures is caused by direct cardiac damage. Elevations of CK-MB are well documented post-procedurally and are related to the incidence of subsequent cardiac events. ${ }^{14}$ Similarly post-procedural elevation of troponin occurs. This can occur due to downstream embolisation from thrombus, atheromatous debris, side branch occlusion, or the effect of periprocedural vessel occlusion. Secondary ischaemic myocardial injury can arise in any situation that will impose a supply-demand mismatch. This may reflect underlying coronary disease. Intense coronary vasoconstriction can accompany cocaine use sufficient to cause myocardial ischaemia and release of

Table 1 Examples of reported elevations of cardiac troponin

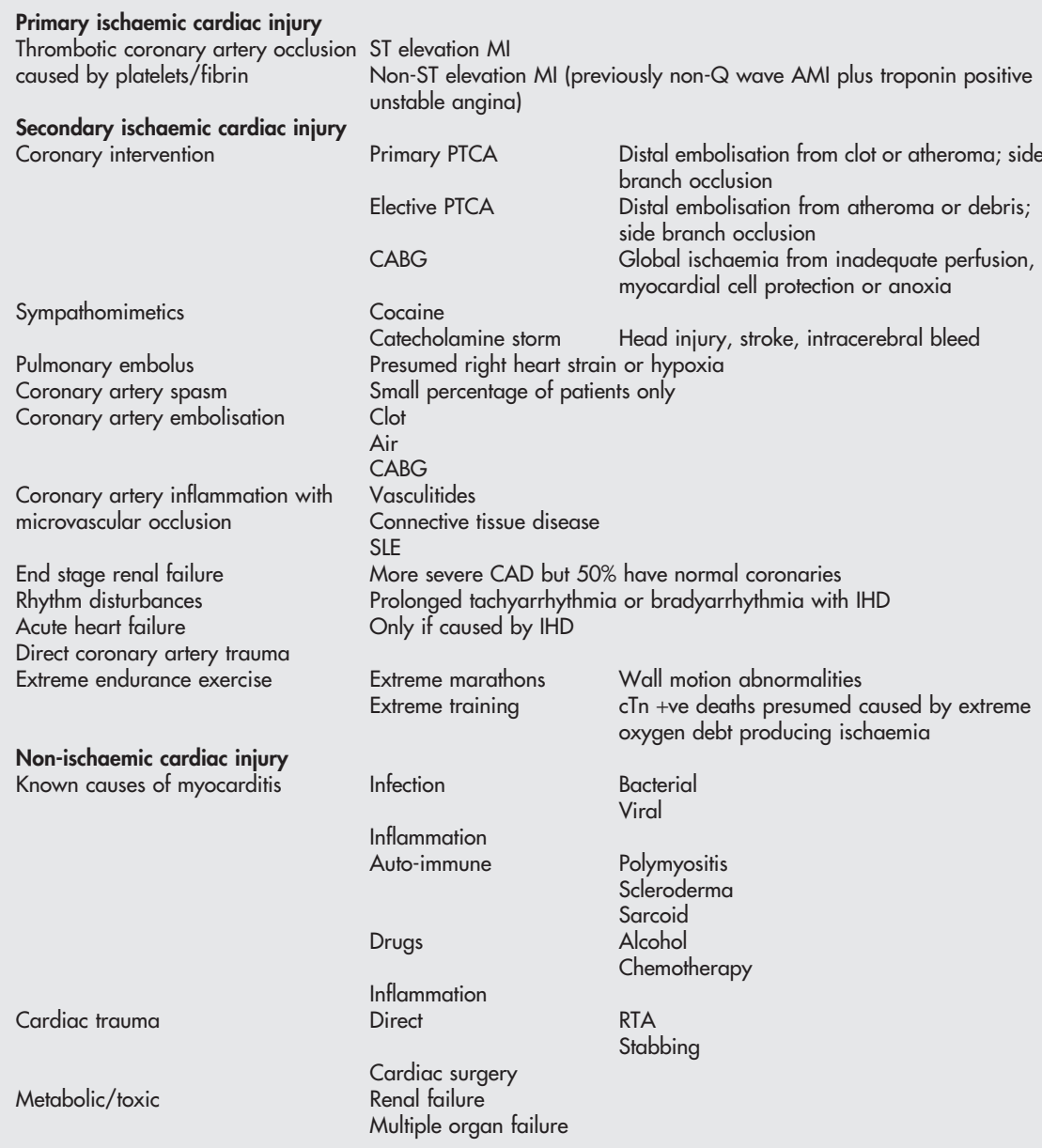

$A M I$, acute myocardial infarction; $C A B G$, coronary artery bypass graft; $C A D$, coronary artery disease; $I H D$, ischaemic heart disease; MI, myocardial infarction; PTCA, percutaneous transluminal coronary angioplasty; RTA, road traffic accident; SLE, systemic lupus erythematosus. 
Table 2 Interpretation of cardiac troponin values

\begin{tabular}{lll}
\hline Troponin value & Comment & Interpretation \\
\hline $\begin{array}{l}\text { Below detection limit of the } \\
\text { assay }\end{array}$ & Undetectable by assay method used & No myocardial necrosis found \\
$\begin{array}{l}\text { Between detection limit and } \\
\text { functional sensitivity } \\
(\% \mathrm{CV} \text { of 10) }\end{array}$ & $\begin{array}{l}\text { Troponin present and can be } \\
\text { distinguished from background but } \\
\text { cannot be quantified repeatably at } \\
\text { this level } \\
\text { Definite myocardial necrosis, } \\
\text { measurements are repeatable }\end{array}$ & $\begin{array}{l}\text { Possible myocardial injury. In the context } \\
\text { of suspected ACS, repeat after } 6 \text { hours } \\
\text { and investigate }\end{array}$ \\
Above functional sensitivity & $\begin{array}{l}\text { AMl when seen in the context of suspected } \\
\text { ACS }\end{array}$ \\
\hline
\end{tabular}

troponin, but the majority of episodes occur when underlying coronary disease is also present. ${ }^{15}$ Whether this is caused by ischaemia and necrosis or ischaemia alone remains to be conclusively proved. The troponin release seen in cerebrovascular events, severe pulmonary infections, and pulmonary embolus fall into this category. The situation in sepsis is less clearly defined but myocardial depression is well described and troponin elevation is an independent mortality predictor. ${ }^{6}$ Direct myocardial damage can occur in any condition where there is direct involvement of the myocardium such as myocarditis or as part of more generalised muscle pathology. Examples are summarised in table 1 .

\section{DIAGNOSTIC CUT OFF}

The recommendations from the ACC/ESC document were for a diagnostic cut off corresponding to the 99th centile of a reference population. Current assays are unable to detect troponin in the majority of normal healthy individuals. This has led to the proposal that an analytical target of percentage coefficient of variation $(\% \mathrm{CV})$ of 10 is used as the cut off for normal. This allows accurate separation of the cardiac and normal populations. The situation may be improved by more sensitive assays that define a true reference population and improve low range assay precision. The majority of assays (but not all) are able to separate a cardiac high risk group from a cardiac low risk group, but improved assay performance has paradoxically increased the number of patients with detectable troponin values who do not have the clinical features of AMI. It may be possible to define, for the general chest pain population, a low level of troponin that does not correspond to a clinically significant degree of risk. The level of troponin that can be reliably measured by the laboratory can be used as the cut point for definite myocardial damage. This corresponds to the functional sensitivity (\%CV of 10 ) for the assay. Levels below this and above the detection limit of the assay indicate possible myocardial damage and should be repeated. In the context of suspected ACS this should trigger further investigations (table 2). Finally, the cardiologist must be aware that different cTnI methods do not always agree and results from bedside testing may not be the same as laboratory testing (and sometimes measure different troponins).

The diagnosis of myocardial infarction remains clinical. A single troponin determination is not sufficient, a minimum of two, and ideally three should be performed to demonstrate changing values. Measurement of cardiac troponin provides a sensitive diagnostic test when used together with other information. The cardiologist must be aware that there are other causes of troponin release and distinguish whether the event is caused by primary plaque rupture requiring intervention or another form of myocardial damage. Clinical assessment of the patient remains essential. The laboratory is an assistant to, not a replacement for, informed clinical decision making.

Are troponins confusing? Not really, unless you stop thinking clinically.

\section{Authors' affiliations}

P O Collinson, Department of Chemical Pathology, St George's Hospital, London, UK

P J Stubbs, Department of Cardiology, Mayday Hospital, Croydon, Surrey, UK

\section{REFERENCES}

1 Collinson PO, Boa FG, Gaze DC. Measurement of cardiac troponins. Ann Clin Biochem 2001;38:423-49.

2 Hamm CW, Ravkilde J, Gerhardt W, et al. The prognostic value of serum troponin T in unstable angina. N Engl J Med 1992;327:146-50.

3 Heller GV, Blaustein AS, Wei JY. Implications of increased myocardial isoenzyme level in the presence of normal serum creatine kinase activity. Am J Cardiol 1983;51:24-7.

4 European Society of Cardiology/American College of Cardiology. Myocardial infarction redefined - a consensus document of the joint European Society of Cardiology/American College of Cardiology committee for the redefinition of myocardial infarction. Eur Heart J 2000;21:1502-13.

5 Bertrand ME, Simoons ML, Fox KA, et al. Management of acute coronary syndromes: acute coronary syndromes without persistent ST segment elevation. Recommendations of the task force of the European Society of Cardiology. Eur Heart J 2000;21:1406-32.

6 Kollef MH, Ladenson JH, Eisenberg PR. Clinically recognized cardiac dysfunction: an independent determinant of mortality among critically ill patients. Is there a role for serial measurement of cardiac troponin I? Chest 1997:111:1340-7.

7 James P, Ellis CJ, Whitlock RM, et al. Relation between troponin T concentration and mortality in patients presenting with an acute stroke: observational study. BMJ 2000;320:1502-4.

8 Haller C, Zehelein J, Remppis A, et al. Cardiac troponin T in patients with endstage renal disease: absence of expression in truncal skeletal muscle [published erratum appears in Clin Chem 1998;44:1586]. Clin Chem 1998;44:930-8.

9 Fredericks S, Murray JF, Bewick M, et al. Cardiac troponin T and creatine kinase $M B$ are not increased in exterior oblique muscle of patients with renal failure. Clin Chem 2001;47:1023-30.

10 Apple FS, Murakami MM, Pearce LA, et al. Predictive value of cardiac troponin I and T for subsequent death in end-stage renal disease. Circulation 2002; 106:2941-5.

11 Giannitsis E, Muller-Bardorff M, Kurowski V, et al. Independent prognostic value of cardiac troponin $\mathrm{T}$ in patients with confirmed pulmonary embolism. Circulation 2000;102:211-7.

12 Davies MJ, Thomas AC, Knapman PA, et al. Intramyocardial platelet aggregation in patients with unstable angina suffering sudden ischemic cardiac death. Circulation 1986:73:418-27.

13 Cannon CP, Weintraub WS, Demopoulos LA, et al. Comparison of early invasive and conservative strategies in patients with unstable coronary syndromes treated with the glycoprotein Ilb/Illa inhibitor tirofiban. NEngl J Med 2001;344:1879-87.

14 Akkerhuis KM, Alexander JH, Tardiff BE, et al. Minor myocardial damage and prognosis: are spontaneous and percutaneous coronary interventionrelated events different? Circulation 2002;105:554-6.

15 Kontos MC, Jesse RL, Tatum JL, et al. Coronary angiographic findings in patients with cocaine-associated chest pain. J.Emerg Med 2003;24:9-13. 